\title{
Neurorehabilitation of the multifaceted and complicated neurologic problems associated with lightning and electrical injuries
}

\author{
Michael Cherington \\ Lightning Data Center, St. Anthony Hospital, 4231 W. 16th Avenue, Denver, CO 80204, USA \\ Tel.: +1 303 629 4258; Fax: +1 303629 4438; E-mail: Lightningdatacenter@Centura.org
}

As a force of pure power and magnificence, nothing in nature surpasses lightning. As a force of importance to the workings and achievements of modern civilization, nothing outranks electricity. Both lightning and electricity, however, can be extremely dangerous and can cause devastating injuries.

In recent years, there have been significant advances in the arenas of trauma and rehabilitation that can apply to the management of lightning and electrical injuries [1,2]. Most medical centers are equipped with both trauma centers and major rehabilitation services.

This issue of NeuroRehabilitation is dedicated to current neurorehabilitation issues of lightning and electrical injuries. The authors are especially competent to write on their topics. This issue contains important information that cannot easily be found in a single publication.

Neurorehabilitation of lightning injuries is essential in the treatment of many, if not most, of these patients. It is surprising, therefore, that there are only a few articles in the literature regarding neurorehabilitation in lightning injuries. As a matter of fact, a PubMed search of this subject yielded only one article solely devoted to this subject. This issue of the journal is dedicated to various neurorehabilitation aspects of this important matter.

By contrast, the medical literature on rehabilitation of electrical injury burns and amputations is sizeable. Severe burns and muscle necrosis are much more likely to occur in generated electrical trauma cases than in lightning patients. The treatment of these severe burns often requires both surgery and rehabilitation.

To open this issue, I present the spectrum of neurologic complications that can tragically ravage those individuals struck by lightning.

The subsequent chapters are written by renowned national and international authorities in the field lightning injuries and electrical trauma.

Daniel Lammertse, an internationally recognized authority on spinal cord injuries, is especially equipped to write the chapter on spinal cord injuries in lightning and electrical trauma. Dan is Medical Director of Craig Hospital, a renowned center for the treatment of spinal cord diseases. Dan is also a member of the Lightning Data Center.

The article on Neurorehabilition of patients with cerebral damage is written by Philip Yarnell, who has extensive experience in treating head trauma and the neurologic complications of lightning/electrical injuries. Phil spent years as Director of Neurology at Denver General Hospital. For twelve years, he has been an active member of the St. Anthony Hospital Lightning Data Center, a referral facility for lightning and electrical trauma cases.

Wolfgang Jost is Chief of the Department of Neurology of the Deutsche Kinik fur Diagnostik in Wiesbaden. He is an authority on the evaluation of autonomic nervous dysfunction in various neurologic dis- 
orders, including Parkinsonism and urogenital disorders. He brings his experience to the article on ANS dysfunction in lightning and electrical trauma patients. ANS dysfunction is responsible for troubling signs and symptoms following lightning/electrical injuries.

Behavioral disorders following lightning and electrical trauma are common and require skilled management. Margaret Primeau is a leading expert in this field. Her article is a must read for all those caring for these often difficult cases.

Electrical burns are particularly devastating. These burns, often accompanied by necrosis of underlying muscle tissue and nerves, require a strong multidisciplinary team of surgeons, anesthesiologists, physiatrists, neurologists, and therapists. Gennaro Selvaggio, plastic surgeon, and his colleagues from the Plastic Surgery Department, Burn Unit of Gent (Belgium) are well situated to write about this subject.

Mary Ann Cooper is a pioneer in the treatment of lightning and electrical injuries. Since her seminal paper in 1980, she has been recognized as an international authority. She and Steve Marshburn, founder of LS\&ESS, Inc. write the article about this unique and important support group for lightning and electrical shock survivors.
Mary Capelli-Schellpfeffer has been a principal investigator in analyzing workplace and industrial electrical injuries. She has written extensively on many aspects of electrical injuries including associated disabilities, prevention and treatments. Her article on roadblocks to return to work after electrical trauma provides an important focus on how to deal with these problems.

Raphael Lee and his colleagues are innovative research leaders who have been pursuing the answers to questions about how lightning and electric current damage human tissues and organs. The chapter by Bier et al. is an elegantly written article on what is known about these biophysical mechanisms including the phenomenon of membrane electroporation.

I believe this issue of NeuroRehabilitation will serve as an extraordinary resource for all those involved in the treatment of lightning and electrical injuries.

\section{References}

[1] A.B. Nathens, F.P. Brunet and R.V. Maier, Development of trauma systems and effect on outcomes after injury, Lancet $\mathbf{3 6 3}$ (2004), 1794-1801.

[2] H. Jackson and D. Manchester, Towards the development of brain injury specialists, NeuroRehabilitation 16 (2001), 27-40. 